\title{
A Experiência de Indexação da Revista Educação \& Formação
}

\author{
NASCIMENTO, Karla Angélica Silva do Nascimento ${ }^{1 *}$ \\ FIALHO, Lia Machado Fiuza ${ }^{\text {***}}$ \\ 1,2 Universidade Estadual do Ceará - Brasil \\ ORCID ID: https://orcid.org/0000-0001-6103-2397* \\ ORCID ID: https://orcid.org/0000-0003-0393-9892**
}

\begin{abstract}
Resumo
A indexação é um processo importante para que as revistas científicas ampliem a divulgação do seu conteúdo. Pensando nisso, o presente estudo tem por objetivo descrever a experiência de indexação da Revista Educação \& Formação (Redufor), durante o processo de solicitação e validação de 20 indexadores, buscadores ou bases de dados. Metodologicamente, utiliza-se o relato de experiência descritivo, que descreve de forma contextualiza e objetiva o levantamento e a vivência durante a indexação da Revista. Foi possível observar que a indexação, na maioria dos casos, não é um processo difícil ou demorado, no entanto, possibilitou a Redufor ampliar sua visibilidade no âmbito nacional e internacional, pois aumentou significativamente o fluxo de publicações de estudos de outros países.
\end{abstract}

Palavras-chave: Indexação. Educação \& Formação. Trabalho Editorial.

\section{The Indexing Experience of Educação \& Formação Journal}

\begin{abstract}
Indexing is an important process for scientific journals to expand the distribution of their content. With that in mind, this study aims to describe the indexing experience at the journal Educação \& Formação (Redufor), during the process of application and approval at 20 indexers, search engines or databases. Methodologically, the descriptive experience report is used, which describes in a contextual and objective way the survey and the experience during the indexing of the Journal. It was possible to observe that, in most cases, the indexing process isn't difficult or time-consuming, however, it allowed Redufor to expand its visibility nationally and internationally, because it significantly increased the influx of foreign studies published.
\end{abstract}

Keywords: Indexing. Education \& Training. Editorial Work.

Título no terceiro idioma: centralizado, em negrito, letras minúsculas e apenas a inicial maiúscula (até 15 palavras)

\begin{abstract}
Resumen
La indexación es un proceso importante para que las revistas científicas amplíen la difusión de su contenido. Teniendo esto en cuenta, este estudio tiene como objetivo describir la experiencia de indexación de Revista Educação \& Formação (Redufor) durante el proceso de solicitud y validación de 20 indexadores, motores de búsqueda o bases de datos. Metodológicamente, se utiliza el relato de experiencia descriptiva, que describe de manera contextual y objetiva la encuesta y la experiencia durante la indexación de la Revista. Se observó que la indexación, en la mayoría de los casos, no es un proceso difícil ni lento, sin embargo, ha permitido a Redufor aumentar su visibilidad a nivel nacional e internacional, ya que ha incrementado significativamente el flujo de publicaciones de estudios en otros países.
\end{abstract}

Palabras clave: Indexación. Educación y Formación. Trabajo Editorial. 


\section{Introdução}

Os serviços de indexação fornecem, aos periódicos, uma cobertura mais ampla para facilitar o acesso aos artigos publicados, especialmente quando estes são publicados em acesso aberto. Nessa direção, este estudo considerou todos os indexadores, bases de dados e buscadores relevantes, ainda que se saiba que a capacidade de cobertura de cada um deles seja diferente, possuindo especificidades particulares.

A indexação de um periódico pode ser considerada, inclusive, um indicador de sua qualidade, tal modo que os periódicos bem indexados são considerados de maior qualidade científica em comparação com os não indexados (FIALHO; SOUSA; FREIRE, 2020). Ante a importância do processo de indexação, importa não apenas conhecer os indexadores, mas a maneira como se solicita ingresso das revistas científicas, o tempo aproximado de avaliação, dentre outros fatores que facilitam o trabalho editorial.

Publicar em revistas de alto impacto é prioridade para muitos pesquisadores e acadêmicos (PACKER, 2011). No entanto, na área das Humanidades e, especificamente, da Educação, campo de atuação da Redufor, objeto de estudo em tela, o "jogo" é desigual. Isso porque os autores encontram dificuldade em publicar seus artigos em revistas de alto impacto já que a especificidade da área não gera índices compatíveis com os de outras áreas, a exemplo da Física e Medicina. Somando-se a isso, os poucos periódicos brasileiros que estão indexados aos grandes órgãos procuram artigos de apelo global, embora percebam que, para esse campo do conhecimento, os artigos majoritariamente possuem foco regionalizado, ensejando ênfase a questões educacionais e sociais que não permitem generalizações (SILVA; DIAS; RIOS, 2020).

Ainda assim, a área da Educação se vê obrigada a considerar o índice de citação na avaliação dos periódicos, optando por utilizar o índice h do Google Scholar para o controle das referências bibliográficas de periódicos acadêmicos, ainda que este muitas vezes não seja realizado de forma qualificada. Existem vários aspectos que podem influenciar o seu cálculo, incluindo cobertura, preferência de idioma do banco de dados, procedimentos mecânicos usados para coletar citações, a não exclusão das autocitações, disponibilidade online de publicações com metadados acessíveis, dentre outros (NASCIMENTO, 2020; MUGNAINI, 2016).

O fato é que apenas seis periódicos brasileiros de Educação estão indexados ao Scopus e nenhum está na coleção principal da Web of Science. Em decorrência, o único cálculo possível de índice de citação para todas as revistas da área de Educação somente é possível por intermédio do Google Scholar. 
Nessa direção, torna-se importante para as revistas científicas aumentar seus índices de citação e, dentre outros fatores, possuir uma boa indexação é fundamental. Com efeito, questionase: Como se pode realizar o processo de indexação de uma revista científica da área de Educação? Para responder a essa problemática, desenvolveu-se um relato de experiência com o objetivo de descrever a experiência de indexação da Revista Educação \& Formação (Redufor), durante o processo de solicitação e validação de 20 indexadores, buscadores ou bases de dados.

Justifica-se a relevância do relato descritivo, pois o mesmo pode servir de subsídio para editores de novos periódicos, já que aponta não apenas indexadores, buscadores ou bases de dados que podem agregar valor à revista científica, mas revela como se dá o acesso a essas fontes de dados, a solicitação de inclusão e o tempo médio de resposta para cada um deles, a exemplo do DOAJ, Dialnet, Index Copernicus, Clase Periódica, Redalyc.org, Latindex, REDIB, Base, Sumários.org, SIS, OAJI.net, ESJI, DRJI, dentre outros.

\section{Metodologia}

A Redufor foi selecionada como objeto de estudo, tanto por ser o campo de atuação das pesquisadoras como pelo fato de ter sido criada em janeiro de 2016 e já possuir mais de 20 indexadores, buscadores ou bases de dados. Essa revista é revisada por pares ${ }^{1}$, está vinculada ao Programa de Pós-Graduação em Educação da Universidade Estadual do Ceará, adota a publicação em fluxo contínuo e publica artigos originais e inéditos, oriundos de pesquisas científicas, na área de Educação. Tem como missão promover o intercâmbio e os debates no campo educacional nacional e internacional, prioritariamente, nos temas "Educação" e "Formação de professores", considerando seu público-alvo: pesquisadores da área da educação, professores da educação básica e superior, pós-graduandos e demais profissionais interessados nos referidos temas (FIALHO, SOUSA, NASCIMENTO, 2020).

Neste estudo, foi necessário, inicialmente, listar os indexadores nacionais e, principalmente de outros países, identificados, uma vez que a Redufor prezou por internacionalizar suas publicações científicas. Em seguida, buscou-se compreender o processo de avaliação de cada indexador devido sua particularidade, seus critérios, políticas e procedimentos para admissão e permanência dos periódicos científicos, representados pelas seguintes coleções: DOAJ, Dialnet, Index Copernicus, Clase Periódica, IRESIE, Redalyc, Amelica, Latindex, REDIB, Base, Sumários.org, Educ@, SIS, OAJI.net, ESJI, DRJI, Scilit, Researchbib, Latinrev Flacso, Journal TOC's.

\footnotetext{
${ }^{1}$ Para refletir sobre revisão por pares sugere-se ler Silva e Mueller (2017). 


\section{Resultados e Discussão}

Diante da identificação prévia, buscou-se formalizar solicitação de indexação por e-mail, bem como, em alguns casos, via preenchimento de formulário eletrônico. Determinados indexadores pediam a apresentação de uma planilha com todos os dados da Revista, incluindo suas últimas publicações em volumes e números. Para detalhar melhor tal procedimento, o próximo item mostra a nacionalidade de cada indexador, caracterizando-o como nacional ou internacional, seu endereço eletrônico, o link de acesso para o instrumento de avaliação ou forma de solicitação e o tempo médio levado receber a confirmação da admissão do periódico, após a solicitação (QUADRO 1).

Quadro 1 - Listagem de indexadores nacionais e internacionais da Redufor

\begin{tabular}{|c|c|c|c|c|}
\hline $\begin{array}{l}\text { INDEXADOR, } \\
\text { BUSCADOR, } \\
\text { BASE DE } \\
\text { DADOS } \\
\end{array}$ & DOMÍNIO & URL & INSTRUMENTO & $\begin{array}{l}\text { CONFIRMAÇÃO } \\
\text { (média em dias) }\end{array}$ \\
\hline DOAJ & Internacional & https://doaj.org/ & $\begin{array}{c}\text { Formulário: } \\
\text { https://doaj.org/application/n } \\
\text { ew }\end{array}$ & 20 \\
\hline Dialnet & Internacional & https://dialnet.unirioja.es/ & $\begin{array}{c}\text { Formulário: } \\
\text { https://dialnet.unirioja.es/serv } \\
\text { let/alta } \\
\end{array}$ & 30 \\
\hline $\begin{array}{l}\text { Index } \\
\text { Copernicus }\end{array}$ & Internacional & $\begin{array}{l}\text { https://www.indexcopern } \\
\text { icus.com/index.php/pl/ }\end{array}$ & $\begin{array}{l}\text { Cadastro no indexador para } \\
\text { ter acesso ao formulário } \\
\text { eletrônico: } \\
\text { https://journals.indexcopernic } \\
\text { us.com/app/auth/login }\end{array}$ & 45 \\
\hline $\begin{array}{l}\text { Clase y } \\
\text { Periódica }\end{array}$ & Internacional & $\begin{array}{l}\text { http://clase.unam.mx/F?f } \\
\text { unc=find-b- } \\
\text { 0\&local_base=cla01 }\end{array}$ & $\begin{array}{l}\text { E-mail para envio de uma } \\
\text { planilha eletrônica com todos } \\
\text { os dados da revista } \\
\text { solicitados: } \\
\text { biblat@ dgb.unam.mx }\end{array}$ & 30 \\
\hline IRESIE & Internacional & $\begin{array}{c}\text { http://iresie.unam.mx/F?f } \\
\text { unc=find-b- } \\
\text { 0\&local_base=irs01 }\end{array}$ & $\begin{array}{c}\text { Envio por e-mail } \\
\text { (biblioteca_iisue@ }{ }^{2} \text { unam.mx) } \\
\text { de um documento de } \\
\text { solicitação: } \\
\text { https://www.iisue.unam.mx/i } \\
\text { resie/formatos/formato_solici } \\
\text { tud_iresie.pdf }\end{array}$ & 15 \\
\hline Redalyc & Internacional & https://www.redalyc.org/ & $\begin{array}{c}\text { Formulário: } \\
\text { https://www.redalyc.org/post } \\
\text { ulacion.oa } \\
\end{array}$ & 20 \\
\hline Amelica & Internacional & $\begin{array}{l}\text { http://amelica.org/index. } \\
\text { php/pt/amelica/ }\end{array}$ & $\begin{array}{c}\text { Formulário: } \\
\text { http://portal.amelica.org/micr } \\
\text { oPortal.oa?opcion=postula }\end{array}$ & 30 \\
\hline Latindex & Internacional & $\begin{array}{l}\text { https://www.latindex.org } \\
\text { /latindex/inicio }\end{array}$ & $\begin{array}{c}\text { Formulário: } \\
\text { https://www.latindex.org/lati } \\
\text { ndex/formReg }\end{array}$ & 20 \\
\hline REDIB & Internacional & https://redib.org/?lng=pt & $\begin{array}{c}\text { Formulário: } \\
\text { https://redib.org/Form/Solicit } \\
\text { udRevista?lng=pt }\end{array}$ & 20 \\
\hline Base & Internacional & $\begin{array}{c}\text { https://www.base- } \\
\text { search.net/ }\end{array}$ & $\begin{array}{c}\text { Formulário: } \\
\text { https://www.base- }\end{array}$ & 15 \\
\hline
\end{tabular}




\begin{tabular}{|c|c|c|c|c|}
\hline & & & $\begin{array}{l}\text { search.net/about/en/contact.p } \\
\text { hp }\end{array}$ & \\
\hline Sumários.org & Nacional & https://sumarios.org/ & $\begin{array}{c}\text { Formulário: } \\
\text { https://sumarios.org/user/regi } \\
\text { ster }\end{array}$ & 5 \\
\hline Educ@ & Nacional & $\begin{array}{c}\text { https://www.fcc.org.br/fc } \\
\text { c/educ }\end{array}$ & $\begin{array}{l}\text { E-mail: educ@fcc.org.br com } \\
\text { envio de planilha eletrônica } \\
\text { específica do órgão com } \\
\text { dados da revista. }\end{array}$ & $* 2$ \\
\hline SIS & Internacional & https://www.sindexs.org/ & $\begin{array}{c}\text { Formulário: } \\
\text { https://www.sindexs.org/Regi } \\
\text { ster.aspx } \\
\end{array}$ & 20 \\
\hline OAJI.net & Internacional & http://oaji.net/ & $\begin{array}{c}\text { Formulário: } \\
\text { http://oaji.net/apply-for- } \\
\text { evaluation-free-service.html }\end{array}$ & 15 \\
\hline ESJI & Internacional & http://esjindex.org/ & $\begin{array}{c}\text { Formulário: } \\
\text { http://esjindex.org/submissio } \\
\text { n.php }\end{array}$ & 10 \\
\hline DRJI & Internacional & http://olddrji.lbp.world/ & $\begin{array}{c}\text { Formulário: } \\
\text { http://olddrji.lbp.world/Sugge } \\
\text { stJournal.aspx } \\
\end{array}$ & 10 \\
\hline Scilit & Internacional & https://www.scilit.net/ & $\begin{array}{c}\text { Formulário: } \\
\text { https://www.scilit.net/registra } \\
\text { tion } \\
\end{array}$ & 10 \\
\hline Researchbib & Internacional & $\begin{array}{l}\text { http://journalseeker.resea } \\
\text { rchbib.com/ }\end{array}$ & $\begin{array}{c}\text { Formulário: } \\
\text { https://www.researchbib.com } \\
\text { /?action=editRegister }\end{array}$ & 20 \\
\hline Latinrev Flacso & Internacional & $\begin{array}{c}\text { https://www.flacso.org.ar } \\
\text { /latinrev/ }\end{array}$ & $\begin{array}{c}\text { E-mail: } \\
\text { redrevistas@ flacso.org.ar }\end{array}$ & 20 \\
\hline Journal TOC's & Internacional & $\begin{array}{c}\text { http://www.journaltocs.a } \\
\text { c.uk/ }\end{array}$ & $\begin{array}{c}\text { E-mail: } \\
\text { journaltocs@hw.ac.uk }\end{array}$ & 15 \\
\hline
\end{tabular}

Fonte: Elaboração das autoras (2020).

Todas as solicitações via e-mail aos indexadores internacionais foram feitas usando o idioma inglês, exceto os hispânicos, ou seja, priorizando a língua espanhola. Assim, é fundamental que o texto de solicitação esteja bem redigido, claro e direto. As informações como: título, endereço eletrônico da revista, ISSN, local e editora, são essenciais para que os indexadores possam conhecer o conteúdo da revista, área de estudo, missão, políticas de acesso aberto e de submissão.

Já os formulários eletrônicos disponibilizados no próprio site do indexador foram preenchidos conforme ordem de obrigatoriedade dos dados. Somente três pediram, além das informações técnicas da revista (Foco, equipe editorial, submissão etc.), as publicações dos últimos três volumes e/ou números, são eles: Index Copernicus, Clase y Periodica e Educ@.

Dos vinte indexadores solicitados, somente o Educ@ ainda não apresentou o resultado de sua avaliação sobre a Redufor. Nesse sentido, não é possível conjecturar o tempo médio de análise, uma vez que a solicitação foi feita em junho de 2019 e após mais de um ano não há qualquer resposta.

\footnotetext{
${ }^{2}$ Até o momento não obtivemos retorno. Dessa forma, não se sabe ao certo quanto tempo a Educ@ dispõe para avaliação do periódico em sua base de dados.
} 
Alguns indexadores, após admissão da Revista em suas bases de dados, solicitaram à equipe editorial o cadastro e atualização de todos as publicações, é o caso da DRJI e Sumários.org. O DOAJ também possui esse procedimento, mas devido comunicação com a plataforma PKP, a atualização, para quem utiliza o OJS, é automática e pode ser feita ao final de cada número. Para isso é necessário selecionar a opção "Ferramentas" do OJS, em seguida o item "Importar/Exportar" e depois em "Exportação para DOAJ". Os demais, utilizam-se das informações e publicações diretamente pelo site da revista, em alguns captados pela interoperabilidade e em outros com o preenchimento manual em suas bases.

\section{Considerações finais}

A partir do problema: como se pode realizar o processo de indexação de uma revista científica da área de Educação?, desenvolveu-se um relato de experiência que teve como objetivo descrever a experiência de indexação da Revista Educação \& Formação (Redufor), durante o processo de solicitação e validação de 20 indexadores, buscadores ou bases de dados.

O relato partiu da identificação de 20 fontes de dados, em seguida mostrou como se efetiva a comunicação com cada uma delas para galgar ingresso/indexação e o tempo médio e resposta desde a experiência da Redufor. Percebeu-se que o processo de solicitação e validação, no caso dos 20 indexadores, bases de dados ou buscadores solicitados é simples e não demora muito tempo para ser efetivado. Constatou-se rapidamente que a Redufor ampliou sua abrangência no que concerne aos acessos, e, em termo de divulgação, a indexação foi positiva, pois possibilitou aumentar o número de artigos publicados de autores nacionais e, especialmente, internacionais. A principal vantagem foi a facilitação do acesso às publicações da Redufor e a sua divulgação em outros países, o que possibilitou triplicar o número de artigos internacionais veiculados e quadruplicar o índice $\mathrm{h}$ do Google Scholar. Sabe-se que a limitação do estudo se dá pela sua impossibilidade de generalização, ademais, sugere-se ampliar o relato de experiência com mais estudos que tratem da indexação em outras coleções mais preteridas como Scopus, Web of Science e SciELO, que se destacam não apenas por gerar métricas, mas pela sua ampla credibilidade no universo editorial.

\section{Referências}

FIALHO, L. M. F.; SOUSA, F. G. A. de; FREIRE, V. C. C. Formação continuada de professores: o que se publica no Norte e Nordeste? Revista Exitus, v. 10, n. 1, p. e020038, 2020. Disponível em: http://www.ufopa.edu.br/portaldeperiodicos/index.php/revistaexitus/article/view/1286. Acesso em: 10 set. 2020 .

FIALHO, L. M. F.; SOUSA, F. G. A. de; NASCIMENTO, K. A. S. do. Educação \& Formação Journal: balance of scientific production (2016-2018). Revista Tempos e Espaços em Educação, v. 13, n. 32, p. 
01-20, 2020. Disponível em: https://seer.ufs.br/index.php/revtee/article/view/12926. Acesso em: 10 set. 2020 .

NASCIMENTO, K. A. S. do. Cenário da produção científica da Revista Educação \& Formação: um estudo bibliométrico. Práticas Educativas, Memórias e Oralidades - Rev. Pemo, Fortaleza, v. 2, n. 1, 2020. Disponível em: https://revistas.uece.br/index.php/revpemo/article/view/3471. Acesso em: 09 set. 2020.

MUGNAINI, R. O Fator de Impacto: sua popularidade, seus impactos e a necessidade de preservação do processo de geração do conhecimento científico. Revista da Escola de Enfermagem da USP, v. 50, n. 5, p. 722-723, 2016. Disponível em: https://www.scielo.br/scielo.php?pid=S0080-

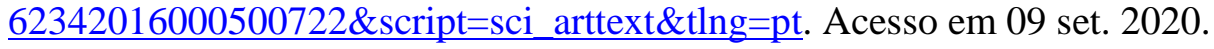

PACKER, A. L. Os periódicos brasileiros e a comunicação da pesquisa nacional. Rev. USP, São Paulo, n. 89, 2011. Disponível em http://rusp.scielo.br/scielo.php?script=sci arttext\&pid=S010399892011000200004\&lng=pt\&nrm=iso. Acessos em 10 set. 2020.

SILVA, I.; DIAS, A.; RIOS, P. Os estudos de Gênero na Revista Tempos e Espaços em Educação: uma Revisão Sistematizada. Educação \& Formação, v. 5, n. 2, p. 150-175, 2020. Disponível em https://revistas.uece.br/index.php/redufor/article/view/2495. Acesso em 10 set. 2020.

SILVA, C. N.; MUELLER, S. A dimensão formativa da revisão por pares em diferentes áreas do conhecimento: prós e contras segundo os laureados da ciência brasileira. Educação \& Formação, v. 2, n. 3, p. 101-122, 2017. Disponível em https://revistas.uece.br/index.php/redufor/article/view/162. Acesso em 10 set. 2020.

\section{${ }^{1}$ Karla Angélica Silva do Nascimento (Fortaleza, Ceará, Brasil)}

Pós-doutoranda em Educação pelo Programa de Pós-Graduação em Educação da Universidade Estadual do Ceará. Doutora em Educação Brasileira pela Universidade Federal do Ceará; Mestra em Educação (Formação de Professores) pela Universidade Estadual do Ceará e graduada em Pedagogia pela Universidade Federal do Ceará, Bolsista PNPD/Capes.

Contribuição de autoria: realizou a escrita, a metodologia, a coleta de dados, copilou os resultados e iniciou as discussões.

Lattes: http://lattes.cnpq.br/5267121220942302

E-mail:karla.asn@gmail.com

\section{${ }^{2}$ Lia Machado Fiuza Fialho (Fortaleza, Ceará, Brasil)}

Professora doutora do Centro de Educação da Universidade Estadual do Ceará, Professora Permanente do Programa de Pós-graduação em Educação (PPGE/UECE). Líder do Grupo de Pesquisa Práticas Educativas Memórias e Oralidades - PEMO. Editora da revista Educação \& Formação do PPGE/UECE. Pesquisadora produtividade CNPQ.

Contribuição de autoria: revisou a escrita, a metodologia e a coleta de dados; contribuiu com os resultados e as discussões, desenvolveu as considerações finais.

Lattes: http://lattes.cnpq.br/4614894191113114

E-mail: lia.fialho@uece.br

Avaliador(a) responsável: Eli Lopes da Silva

\section{Como citar este artigo:}

NASCIMENTO, Karla Angélica Silva do, FIALHO, Lia Machado Fiuza. A Experiência de Indexação da Revista Educação \& Formação. In: ABEC Meeting Live, 2020. Anais... São Paulo: Associação Brasileira de Editores Científicos, 2020. DOI: http://dx.doi.org/10.21452/abecmeeting2020.09 